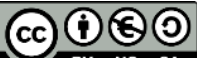

\title{
Marketing Publicitario Mipymes.
}

\author{
Marketing advertising Mipymes.
}

Velasteguí López Efraín. ${ }^{1}$, Guamangate Chiguano Marco. ${ }^{2}$

DOI: $\underline{\text { https://doi.org/10.33262/visionariodigital.v1i3.255 }}$

\section{Resumen.}

El trabajo investigativo se orientó al diseño de un plan de marketing para mejorar la gestión de ventas mediante la aplicación de estrategias de marketing, publicidad, promoción e implementación de un plan de marketing el desarrollo de la investigación y el avance de la tecnología ha permitido el crecimiento en distintas áreas del conocimiento como la medicina, ingeniería, educación; entre otras de las cuales se resalta en entorno web que ha revolucionado el ámbito empresarios actualizado la forma de hacer negocios de manera presencial a distintas a través de medios de comunicaciones que favorecen la interacción entre empresas y mercado llegando a más clientes las existencia de la sociedad contemporánea denotan una necesidad imperiosa de las pymes a incursionaren el mundo informática para explorar sus potencialidades con la finalidad de crear un volar agregado a toda organización que marque la diferencias entre el éxito y el fracaso; entre los medios se cita a los redes sociales debido a que son las más usadas gracias a que poseen una mayor versatilidad que facilita la empresa realizar acciones remotas como darse a conocer de forma indirecta una estrategia de marketing que fomente al desarrollo empresarial de las pymes en el ecuador para fundamentar el estudio se realizó esta investigación buscar dar a conocer las virtudes que la redes prestan a las empresas en el entorno socioeconómico del ecuador las pymes son todas pequeñas y medianas empresas que se dedican a verde sus productos o presta servicios a cambio de remuneración en una empresa capaz de influir en la economía local o nacional.

Palabras clave: Marketing, publicitario, pequeña empresa, Mipymes.

\footnotetext{
${ }^{1}$ Universidad Técnica de Ambato, Ambato, Ecuador, le.velastegui@uta.edu.ec

${ }^{2}$ Universidad Técnica de Cotopaxi, Cotopaxi, Ecuador, guamangat23@gmail.com
} 
Abstract.

The research work was oriented to the design of a marketing plan to improve sales management through the application of marketing strategies, advertising, promotion and implementation of a marketing plan. The development of research and the advancement of technology has allowed the growth in different areas of knowledge such as medicine, engineering, education; among others, it stands out in a web environment that has revolutionized the business sector updated the way of doing business in person to different through communication means that favor the interaction between companies and market reaching more customers the existence of society contemporary denote a compelling need for SMEs to venture into the computer world to explore their potential in order to create a fly added to any organization that marks the differences between success and failure; Among the media, social networks are cited because they are the most used because they have a greater versatility that facilitates the company to perform remote actions such as indirectly disclosing a marketing strategy that encourages the business development of SMEs in The Ecuador to support the study was conducted this research seek to publicize the virtues that networks provide to companies in the socioeconomic environment of Ecuador SMEs are all small and medium enterprises that are dedicated to green their products or provide services in exchange for remuneration in a company capable of influencing the local or national economy.

Keywords: Marketing, advertising, Mipymes.

\section{Introducción.}

La presente investigación diseño de (Juan, 2013; Daniel, 2009 y Ricardo 2010) un plan marketing para mejorar la gestión ventas es relevante para la empresa en estudios considerando que es necesario para el buen funcionamiento mediante la comercialización eficaz y rentable de los productos ofertados en el mercado.

El plan marketing es un documento escrito que detalla la situación actual respeto de los clientes, competidores y ambientales externo, el cual proporciona las pautas para las asignaciones de objetivos, acciones de marketing y recursos a lo largo del periodo de planeación para un producto o servicio existente o presupuesto. Los métodos utilizados en el desarrollo del trabajo investigativo fueron: deductivo, el cual se empleó en el estudio de tipos y estrategias del plan marketing para determinar la gestión de publicidad en los almacenes, así también el método analítico, mediante estudios de cada uno de sus elementos y las relaciones entre los mismo, destacándose la administración como parte para mejorar el área de gestión de publicidad.

Está enfocado en realizar un análisis crítico de todos los aspectos relacionados con la comercialización de la empresa en el mercado. El objetivo general del trabajo fue plasmar en un documento una herramienta útil, que le permita a los almacenes realizar sus publicidad, mejorar sustentablemente los volúmenes de ventas, posesionar su marca en la 
mente de los consumidores y sobre todo satisfacer las necesidades insatisfechas del mercado, a través, de un servicio enmarcado en los altos estándares de calidad.

Para la cual, se estimó procedente realizar un análisis situacional, a fin de analizar e identificar los factores que inciden directamente en el medio donde la empresa desarrolla la actividad comercial, logrando evaluar uno a uno los posibles acontecimientos hasta llegar a determinar fortalezas, amenazas, debilidades y oportunidades, que influyen directamente en el desarrollo del negocio. Además, se desarrolló un estudio de mercado, para lo cual se utilizó encuentras dirigidas a clientes internos y externos de la empresa, con el fin de evidenciar las necesidades del mercado y sobre todo identificar las falencias de la organización, que justifique la necesidad de implementar el plan de marketing a fin de mejorar la calidad del servicio enfocado en posicionar la marca y sobre todo aumentar el volumen de ventas para realizar la publicidad.

Luego la investigación se encamino a plantear una serie de estrategias y técnicas de comercialización enfocadas al fortalecimiento de la calidad del servicios, promoción y publicidad del almacén, a fin de incentivar la adquisición de los productos, logrando cumplir de esta manera con unos de los objetivos del plan marketing que es elevar el nivel sostenible de ventas mediantes la promoción de los productos. Finalmente se realizó el análisis financiero, el mismo que permitió determinar técnicamente los beneficios económicos que generara la implementación del plan de marketing, asegurando de esta manera el aprovechamiento de las oportunidades comerciales de las empresas.

El marketing es el conjunto de actividades y recursos puestos en la práctica para detectar una necesidad, desarrollar el producto que la satisface y hacerlo llegar hasta el consumidor. 
Imagen N 1. Datos estadísticos de marketing publicitario.

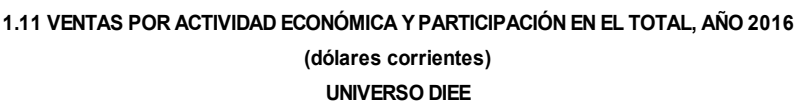

\begin{tabular}{|c|c|c|c|}
\hline Actividad Económica & \multicolumn{2}{|c|}{ Ventas Totales } & \multirow{2}{*}{$\begin{aligned} & \% \text { Total } \\
& 100,0 \% \\
&\end{aligned}$} \\
\hline TOTAL & $\$$ & 147.729.572.739 & \\
\hline COMERCIO AL POR MAYOR Y AL POR MENOR; REPARACIÓN DE VEHÍCULOS AUTOMOTORES Y MOTOCICLETAS. & $\$$ & 55.268 .966 .243 & $37,4 \%$ \\
\hline INDUSTRIAS MANUFACTURERAS. & $\$$ & 30.111 .337 .513 & $20,4 \%$ \\
\hline EXPLOTACIÓN DE MINAS Y CANTERAS. & $\$$ & 10.881 .888 .565 & $7,4 \%$ \\
\hline AGRICULTURA, GANADERÍA, SILVICULTURA Y PESCA. & $\$$ & 7.873 .752 .279 & $5,3 \%$ \\
\hline ACTIVIDADES FINANCIERAS Y DE SEGUROS. & $\$$ & 7.388.913.154 & $5,0 \%$ \\
\hline CONSTRUCCIÓN. & $\$$ & 6.193 .941 .101 & $4,2 \%$ \\
\hline TRANSPORTE Y ALMACENAMIENTO. & $\$$ & 5.793 .300 .926 & $3,9 \%$ \\
\hline INFORMACIÓN Y COMUNICACIÓN. & $\$$ & 5.199 .324 .357 & $3,5 \%$ \\
\hline ACTIVIDADES PROFESIONALES, CIENTÍFICAS Y TÉCNICAS. & $\$$ & 4.389 .996 .469 & $3,0 \%$ \\
\hline SUMINISTRO DE ELECTRICIDAD, GAS, VAPOR Y AIRE ACONDICIONADO. & $\$$ & 3.192 .101 .065 & $2,2 \%$ \\
\hline ACTIVIDADES DE SERVICIOS ADMINISTRATIVOS Y DE APOYO. & $\$$ & 2.558 .804 .245 & $1,7 \%$ \\
\hline ACTIVIDADES DE ATENCIÓN DE LA SALUD HUMANA Y DE ASISTENCIA SOCIAL. & $\$$ & 1.886 .750 .347 & $1,3 \%$ \\
\hline ENSEÑANZA. & $\$$ & 1.882 .442 .066 & $1,3 \%$ \\
\hline ACTIVIDADES DE ALOJAMIENTO Y DE SERVICIO DE COMIDAS. & $\$$ & 1.870 .653 .608 & $1,3 \%$ \\
\hline ACTIVIDADES INMOBILIARIAS. & $\$$ & 1.555 .358 .275 & $1,1 \%$ \\
\hline OTRAS ACTIVIDADES DE SERVICIOS. & $\$$ & 778.396 .673 & $0,5 \%$ \\
\hline DISTRIBUCIÓN DE AGUA; ALCANTARILLADO, GESTIÓN DE DESECHOS Y ACTIVIDADES DE SANEAMIENTO. & $\$$ & 639.062 .186 & $0,4 \%$ \\
\hline ARTES, ENTRETENIMIENTO Y RECREACIÓN. & $\$$ & 258.835 .471 & $0,2 \%$ \\
\hline ADMINISTRACIÓN PÚBLICA Y DEFENSA; PLANES DE SEGURIDAD SOCIAL DE AFILIACIÓN OBLIGATORIA. & $\$$ & 5.748 .196 & $0,0 \%$ \\
\hline
\end{tabular}

Fuente: http://www.ecuadorencifras.gob.ec/estadisticas-de-las-empresas/

Elabora por: Directorio de empresa - DIEE 
Imagen N 2. Datos estadísticos de marketing publicitario.

1.12 VENTAS POR PROVINCIA Y PARTICIPACIÓN NACIONAL, AÑO 2016

(dólares corrientes)

UNIVERSO DIEE

\begin{tabular}{|c|c|c|c|}
\hline Provincia & \multicolumn{2}{|c|}{ Ventas Totales } & \% Total Ventas \\
\hline TOTAL & $\$$ & 147.729 .572 .739 & $100,0 \%$ \\
\hline PICHINCHA & $\$$ & 66.134.160.699 & $44,8 \%$ \\
\hline GUAYAS & $\$$ & 48.173.946.069 & $32,6 \%$ \\
\hline AZUAY & $\$$ & 7.515.105.106 & $5,1 \%$ \\
\hline MANABÍ & $\$$ & 4.847 .290 .190 & $3,3 \%$ \\
\hline EL ORO & $\$$ & 4.399 .218 .336 & $3,0 \%$ \\
\hline TUNGURAHUA & $\$$ & 2.973 .637 .773 & $2,0 \%$ \\
\hline LOS RÍOS & $\$$ & 2.225 .683 .272 & $1,5 \%$ \\
\hline SANTO DOMINGO DE LOS TSÁCHILAS & $\$$ & 2.006 .941 .105 & $1,4 \%$ \\
\hline ESMERALDAS & $\$$ & 1.410 .997 .556 & $1,0 \%$ \\
\hline IMBABURA & $\$$ & 1.392 .652 .276 & $0,9 \%$ \\
\hline LOJA & $\$$ & 1.226 .914 .492 & $0,8 \%$ \\
\hline СОTOPAXI & $\$$ & 1.145 .590 .626 & $0,8 \%$ \\
\hline CHIMBORAZO & $\$$ & 1.044 .523 .234 & $0,7 \%$ \\
\hline SANTA ELENA & $\$$ & 683.111 .183 & $0,5 \%$ \\
\hline CAÑAR & $\$$ & 553.229 .241 & $0,4 \%$ \\
\hline SUCUMBíOS & $\$$ & 408.021 .173 & $0,3 \%$ \\
\hline CARCHI & $\$$ & 351.469 .981 & $0,2 \%$ \\
\hline ORELLANA & $\$$ & 344.662 .206 & $0,2 \%$ \\
\hline GALÁPAGOS & $\$$ & 219.894 .180 & $0,1 \%$ \\
\hline PASTAZA & $\$$ & 153.181 .434 & $0,1 \%$ \\
\hline BOLÍVAR & $\$$ & 139.948 .805 & $0,1 \%$ \\
\hline MORONA SANTIAGO & $\$$ & 130.833 .808 & $0,1 \%$ \\
\hline NAPO & $\$$ & 130.605 .965 & $0,1 \%$ \\
\hline ZAMORA CHINCHIPE & $\$$ & 117.954 .029 & $0,1 \%$ \\
\hline ZONA NO DELIMITADA & $\$$ & & $0,0 \%$ \\
\hline
\end{tabular}

Fuente: http://www.ecuadorencifras.gob.ec/estadisticas-de-las-empresas/

Elabora por: Directorio de empresa - DIEE 
Imagen $\mathbf{N}^{\circ}$ 3. Datos estadísticos de marketing publicitario.

1.13 EVOLUCIÓN DE PERSONAL AFILIADO EN EL PERÍODO 2006 - 2016 UNIVERSO DIEE

\begin{tabular}{|l|r|r|r|}
\hline Año & Afiliados Totales & Afiliados Hombres & \multicolumn{1}{c|}{ Afiliados Mujeres } \\
\hline 2006 & 1.363 .488 & 851.456 & 512.032 \\
\hline 2007 & 1.475 .316 & 919.215 & 556.101 \\
\hline 2008 & 1.680 .504 & 1.053 .019 & 627.485 \\
\hline 2009 & 1.831 .478 & 1.143 .975 & 687.503 \\
\hline 2010 & 2.061 .809 & 1.284 .147 & 777.662 \\
\hline 2011 & 2.418 .628 & 1.511 .899 & 906.729 \\
\hline 2012 & 2.727 .384 & 1.695 .094 & 1.032 .290 \\
\hline 2013 & 2.941 .782 & 1.820 .120 & 1.121 .662 \\
\hline 2014 & 3.090 .034 & 1.905 .415 & 1.184 .619 \\
\hline 2015 & 3.092 .720 & 1.900 .968 & 1.191 .752 \\
\hline 2016 & 2.937 .554 & 1.788 .476 & 1.149 .077 \\
\hline
\end{tabular}

Fuente: http://www.ecuadorencifras.gob.ec/estadisticas-de-las-empresas/

Elabora por: Directorio de empresa - DIEE

Imagen $\mathbf{N}^{\circ}$ 4. Datos estadísticos de marketing publicitario.

1.16 EVOLUCIÓN DE LA MASA SALARIAL EN EL PERÍODO 2006 - 2016

(dólares corrientes) UNIVERSO DIEE

\begin{tabular}{|l|lr|}
\hline Año & \multicolumn{2}{|c|}{ Masa Salarial } \\
\hline 2006 & $\$$ & 5.197 .782 .029 \\
\hline 2007 & $\$$ & 6.252 .963 .045 \\
\hline 2008 & $\$$ & 8.156 .707 .701 \\
\hline 2009 & $\$$ & 10.125 .336 .750 \\
\hline 2010 & $\$$ & 12.250 .147 .674 \\
\hline 2011 & $\$$ & 15.588 .489 .858 \\
\hline 2012 & $\$$ & 18.641 .758 .203 \\
\hline 2013 & $\$$ & 21.414 .475 .222 \\
\hline 2014 & $\$$ & 23.798 .334 .784 \\
\hline 2015 & $\$$ & 24.897 .398 .257 \\
\hline 2016 & $\$$ & 24.825 .237 .545 \\
\hline
\end{tabular}

Fuente: http://www.ecuadorencifras.gob.ec/estadisticas-de-las-empresas/ Elabora por: Directorio de empresa - DIEE 
www.visionariodigital.org

Imagen $\mathbf{N}^{\circ}$ 4. Datos estadísticos de marketing publicitario.

1.17 MASA SALARIAL POR ACTIVIDAD ECONÓMICA Y PARTICIPACIÓN EN EL TOTAL, AÑO 2016

(dólares corrientes)

UNIVERSO DIEE

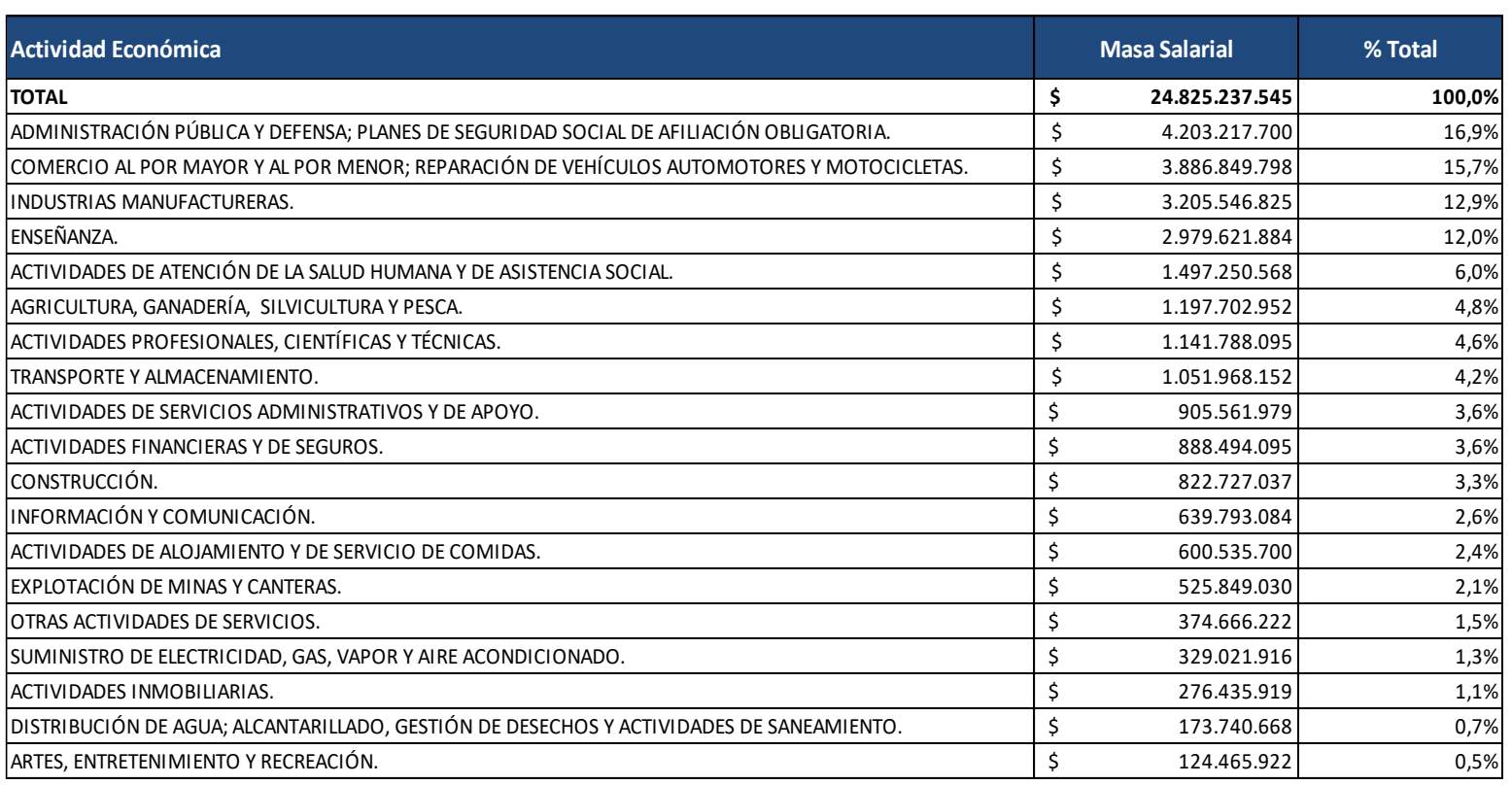

Fuente: http://www.ecuadorencifras.gob.ec/estadisticas-de-las-empresas/

Elabora por: Directorio de empresa - DIEE

Imagen N 4. Datos estadísticos de marketing publicitario.

1.21 VENTAS POR ACTIVIDAD ECONÓMICA Y PARTICIPACIÓN EN EL TOTAL, AÑO 2016

(dólares corrientes)

EMPRESAS CON ACTIVIDADES PRODUCTIVAS

\begin{tabular}{|c|c|c|c|}
\hline Actividad Económica & \multicolumn{2}{|c|}{ Ventas Totales } & $\%$ Total \\
\hline TOTAL & $\$$ & 139.906.218.158 & $100,0 \%$ \\
\hline COMERCIO AL POR MAYOR Y AL POR MENOR; REPARACIÓN DE VEHÍCULOS AUTOMOTORES Y MOTOCICLETAS. & $\$$ & 53.514 .186 .293 & $38,3 \%$ \\
\hline INDUSTRIAS MANUFACTURERAS. & $\$$ & 29.980 .785 .066 & $21,4 \%$ \\
\hline EXPLOTACIÓN DE MINAS Y CANTERAS. & $\$$ & 10.585.761.169 & $7,6 \%$ \\
\hline AGRICULTURA, GANADERÍA, SILVICULTURA Y PESCA. & $\$$ & 7.609 .943 .856 & $5,4 \%$ \\
\hline ACTIVIDADES FINANCIERAS Y DE SEGUROS. & $\$$ & 7.165 .842 .605 & $5,1 \%$ \\
\hline CONSTRUCCIÓN. & $\$$ & 5.916 .521 .239 & $4,2 \%$ \\
\hline TRANSPORTE Y ALMACENAMIENTO. & $\$$ & 5.484 .287 .488 & $3,9 \%$ \\
\hline INFORMACIÓN Y COMUNICACIÓN. & $\$$ & 5.157 .817 .895 & $3,7 \%$ \\
\hline ACTIVIDADES PROFESIONALES, CIENTÍFICAS Y TÉCNICAS. & $\$$ & 4.104 .749 .922 & $2,9 \%$ \\
\hline SUMINISTRO DE ELECTRICIDAD, GAS, VAPOR Y AIRE ACONDICIONADO. & $\$$ & 3.185 .020 .635 & $2,3 \%$ \\
\hline ACTIVIDADES DE SERVICIOS ADMINISTRATIVOS Y DE APOYO. & $\$$ & 2.514 .095 .018 & $1,8 \%$ \\
\hline ACTIVIDADES DE ALOJAMIENTO Y DE SERVICIO DE COMIDAS. & $\$$ & 1.848 .184 .768 & $1,3 \%$ \\
\hline ACTIVIDADES INMOBILIARIAS. & $\$$ & 1.244 .904 .834 & $0,9 \%$ \\
\hline OTRAS ACTIVIDADES DE SERVICIOS. & $\$$ & 717.966 .546 & $0,5 \%$ \\
\hline DISTRIBUCIÓN DE AGUA; ALCANTARILLADO, GESTIÓN DE DESECHOS Y ACTIVIDADES DE SANEAMIENTO. & $\$$ & 629.331 .995 & $0,4 \%$ \\
\hline ARTES, ENTRETENIMIENTO Y RECREACIÓN. & $\$$ & 246.818 .829 & $0,2 \%$ \\
\hline
\end{tabular}

Fuente: http://www.ecuadorencifras.gob.ec/estadisticas-de-las-empresas/

Elabora por: Directorio de empresa - DIEE 
La implementación de (María, 2011) las nuevas tecnologías ocasiona un es fuerzo importante para las empresas. Así, la innovación supone un elevado desembolso económico para las pymes, que debe ser enfocado como una inversión, la mejora del producto al ver aumentada su calidad, logra una mejora del producto, al ver aumentada su calidad, logra una mejora competitiva. También supone una inversión en formación de los empleados de manera que se adapten a los nuevos métodos de trabajo y gestión de la empresa, sea mayor la transferencia de conocimiento y pueda ser implementadas y plenamente utilizadas.

Las existencias de empresas líderes en la utilización de nuevas tecnologías, puede suponer una mayor flexibilidad y ventaja en la difusión de las innovaciones tecnológicas dentro de la economía. Las grandes diferentes entre las grandes empresas que utilizan intensivamente las nuevas tecnologías y las pequeñas empresas ancladas en muchos casos en métodos y sistema obsoletos, tiene un efecto positivo sobre la eficiencia para que reduzcan así su desventajas competitiva, por otra, expulsados del mercado a las empresas lentas o incapaces de introducir estas innovaciones.

En una investigación teórica realizada de (Cesar, 2010; Roberto, 2012; Flavio, 2009 y Claudia 2013) complementariedad entre capital humana y tecnología ha sido objeto asimismo de numeras contrastaciones empíricas que confirman que a mayor calidad de los recursos humanos más innovadores son las empresas y mayor productividad tienes. En efecto, puesto que toda la transmisión de información o de conocimientos requiere, para que sea realmente fructífera, un receptor capaz de absorber y aprovechar lo que se le transmite, cuando mayores sean las capacidades de los empleados, mayores son las posibilidades de conseguir mejores productividad.

Una empresa sea líder o este rezagada en su nivel de adopción de tics dependerá de sus percepciones acerca de los costos y beneficios de tal decisión, la naturaleza y numero de las transacciones que realice en sus actividad, así como su deseo de dar este salto tomando en cuenta su tamaño, las características de su dueño o administrador el número de proveedores y consumidores y la posibilidad real de sus proveedores/consumidores de utilizar la internet como mecanismo para realizar transacciones comerciales.

La decisión de una Mipyme para tratar de alcanzar un alto nivel de adaptación de tics es el resultado de muchos factores. Estos factores no solo incluyen el tradicional análisis costo beneficio de una decisión de inversión. Sino la complejidad de la tecnología y el grado de preparación de la red de consumidores y proveedores.

Existe muchas imprecisiones (Charles, 2009; José, 2012; Jiménez, 2010 y Ramírez 2013) generalmente se las asocia como entes informales, como empresas de subsistencia y de tipo familiar como las pequeñas unidades tales como la tienda de la esquina la panadería, el puesto del mercado. Hasta hoy no hay una categorización regida para la determinar que es una pyme no existe uniformidad entre países; cada cual elige el parámetro que se adapta a su realidad además observamos que los criterios o parámetros para clasificar a las pymes varían de uno u otro países y que incluso los elementos de 
categorización son distintas y de aplicación diversas, así se usa indistintamente para su clasificación los conceptos de ventas, números e empleados y nivel de activos dentro de este amplio universo y no haciendo una caracterización única, tales parámetros, aplicando en distintos países y realidad permitiendo englobar a muchos empresas o unidades más de la citado en el párrafo anterior, agregaríamos el emprendimiento, empresas de nicho de alfa tecnología, empresas exploradas e incluso, para defectos del ranking de esta unidades productivas.

Tabla 1. Ventajas y desventajas de marketing publicitario.

\begin{tabular}{|c|c|}
\hline Ventajas & Desventajas \\
\hline Están más cerca de sus clientes. & Tienen más dificultades para financiarse. \\
\hline Son más flexibles. & $\begin{array}{l}\text { Puede resultarles difícil llegar a un gran número de } \\
\text { clientes y ganarse su confianza. }\end{array}$ \\
\hline $\begin{array}{l}\text { Tienen mayor facilidad para detectar y aprovechar } \\
\text { pequeños nichos de mercado. }\end{array}$ & Los costes son más altos. \\
\hline Son más rápidas tomando decisiones. & No resulta fácil soportar crisis prolongadas. \\
\hline Será más fácil vincular al personal con la empresa. & $\begin{array}{l}\text { Escaso poder de negociación con proveedores y } \\
\text { clientes. }\end{array}$ \\
\hline Todo el mundo se conoce. & Acceso a personal menos capacitado. \\
\hline La comunicación será más sencilla. & $\begin{array}{l}\text { Tendrán más dificultades para acceder a } \\
\text { tecnología. }\end{array}$ \\
\hline
\end{tabular}

Es la función comercial que identifican las necesidades y los deseos de los clientes, determinan que mercados meta pueden atender mejor la organización, y diseña productor, servicios y programas apropiados para atender a esos mercados. Sin embargo, el marketing es mucho más que una función aislada de los negocios es una filosofía que guía a toda la organización. La meta del marketing es crear satisfacción de los clientes de forma rentable, forjando relaciones cargadas de valor con los clientes importantes. El departamento de marketing no puede alcanzar este objetivo por sí solo. Los avances científicos y tecnológicos de los últimos años, así como los cambios políticos, sociales culturales y económicos tanto e ecuador como en otras latitudes han exigido que los individuos se capaciten para mejorar sus habilidades y destrezas. En ciudades ecuatorianas como Riobamba, la falta de inversión en programas de capacitación para el personal de empresas, organizaciones e instituciones, ha generado que las personas por cuenta propia busquen constantemente mejorar, actualizar y profundizar sus conocimientos en temas de interés, frente a una sociedad tendiente a la globalización y cada vez más orientada a la búsqueda de profesionales competitivos en el ámbito laboral.

El contexto de la provincia de Cotopaxi e la particular del cantón es totalmente deferente, pues no se cuentan con centros de contabilidad y auditoría que permita mejorar la situación actual que presenten los directivos y empresarios de la empresas en el cantón en materia contable. La idea se sustenta bajo oportunidades identificadas en el entorno, una de ellas es la necesidad que hoy en día la persona de capacitarse constantemente dado que le mercado laboral actualmente demanda de profesiones competentes además que la 
ciencia y la tecnología avanza y es necesario constantemente actualizar y profundizar nuestros conocimientos en áreas que generan gran demanda en el mercado, puesto que la aplicación d estos conocimientos sirve para gerencia eficiencias el futuro de cualquier empresa o negocio.

La literatura comercialización es amplia y refrenda las esencias de un proceso complejo y dinámico. La comercialización es la acción y efecto de comercializar poner en venta un producto o darle las condiciones y vías de distribuciones para su venta. La noción de comercialización tiene distintos usos según el contexto. Es posibles asociar la comercialización tiene distintos usos según el contexto. Es posibles asociar la comercialización a la distribución o logística que se encarga de hacer llegar físicamente el producto o el servicio al consumidor final. El objetivo de la comercialización, en este sentido, es ofrecer el producto en el lugar y momento en que el consumidor desea adquirir. La comercialización comprende un procedimiento que el cliente general no ve en su totalidad, es más, el comprador es la parte final del proceso, este proceso antes del referido trueque final tiene que ser evaluado, analizado para sí determinar el campo en el que deber ser implantado el proceso de compra y venta. En este importancia análisis se toman en cuentan muchas variables importantes. Significa que una organización encamina todos sus esfuerzos a satisfacer a sus clientes por una ganancia.

Basado en los conceptos de (Escudero \& Herrera, 2014) antes mencionados se concluyó que la comercialización es la actividad como tal que se realiza en el comercio. Es el intercambio que se aplica cuando una persona quiere adquirir un producto. Se ocupa de aquello que los clientes desean, y sirve de guía para lo que se produce y se ofrece. La comercialización es a la vez un conjunto de actividades realizadas por organizaciones, y un proceso social. Se da en dos planos, micro y macro. Se utilizan dos definiciones: micro comercialización y macro comercialización. Significa que una organización encamina todos sus esfuerzos a satisfacer a sus clientes por una ganancia y se concreta a través de canales de comercialización, actualmente las empresas se enfrentan a un ambiente competitivo y exigencias por parte de los consumidores de cual genera inestabilidad y un mayor desafío de implementar planes y estrategias para asegurar la estabilidad y permanencia en el mercado; es así como surge la necesidad del diseño de un plan marketing como una herramienta empresarial para lograr un mejor posicionamiento en el mercado.

El presente trabajo de investigativo tiene como finalidad determinar las estrategias de mercado que permita al comercial, posicionarse como líder del mercado, mediante la implementación de un plan de marketing se creara una variedad de instrumentos que permitan a la empresa generar valor agregado frente a la competencia logrando alcanzar mayores ventas y por ende mejorar la rentabilidad y posicionamiento de la institución. El problema principal que afronta el comercio, es la carencia de un plan de marketing que permita conocer las preferencias y necesidades de sus clientes, impulsando a mejorar sus posiciones, marca y servicio de venta, que permita el desarrollo continuo de la entidad. 
En el ecuador el plan de marketing de (María \& Pérez, 2014) se ha utilizado como una herramienta indispensable en la consecución de los objetivos propuestos por las empresas medianas y grandes, porque se ha logrado evidenciar resultados positivos permitiendo posicionar al producto o servicio en la mentalidad de los consumidores, la actividad comercial ha crecido de manera acelerada en la provincia de Cotopaxi lo cual ha generado una competencia feroz por captar la atención de los clientes para así incrementar el volumen de la ventas.

Una cultura de administración tradicional donde los planes de marketing son una herramienta desconocida y poco utilizada por parte de los directivos de las empresas. Comerciales es un ente dedicado a la comercialización de electrodoméstico que carece de un plan de marketing que permita aprovechar las potencialidades de sus recursos para lograr un posicionamiento entre aprovechar las potencialidades de sus recursos para lograr u posicionamiento entre la ciudadanía logrando sobresalir en un entorno cambiante que evoluciona con el transcurso de los años.

Imagen N 1. Datos estadísticos de marketing Mipymes .

Composición de los ingresos de las empresas medianas por sector

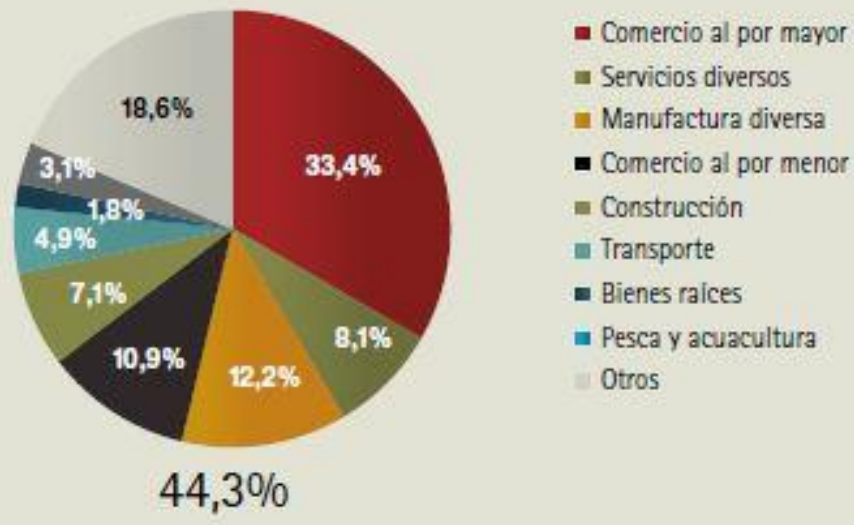

Fuente: http://www.ekosnegocios.com/negocios/verArticulo?idArt=1229 Elabora por: SRI

En esta investigación de (Esteba \& Arturo, 2014) enfocado en realizar un análisis crítico de todos los aspectos relacionados con el comercialización de la empresa en el mercado. El objetivo general del trabajo fue plasmar en un documento una herramienta útil, que le permita a los almacenes de electrodomésticos mejorar sustentablemente los volúmenes de ventas, posicionar su marca en la mente de los consumidores y sobre todo satisfacer las necesidades insatisfechas del mercado, a través de un servicio enmarcado en los más altos estándares de calidad. Para lo cual, se emitió procedente realizar un análisis situacional a fin de analizar e identificar los factores que inciden directamente en el medio 
donde la empresa desarrolla la actividad comercial, logrando evaluar uno a uno los posibles acontecimientos hasta llegar a determinar fortalezas, amenazas, debilidades $\mathrm{u}$ oportunidades, que influyen directamente con el desarrollo del negocio. Además, se desarrolló un estudio de mercado, para lo cual se utilizó encuentras dirigidas al cliente internos y externos de la empresa, con el fin de evidenciar las necesidades del mercado y sobre todo identificar las falencias de la organización que justifiquen la necesidad de implementar el plan de marketing a fin de mejorar la calidad del servicios enfocado en posicionar la marca y sobre todo aumentar el volumen de ventas.

Luego la investigación se encamino a plantear una serie de estrategias y técnicas de comercialización enfocadas al fortalecimiento de la calidad del servicio, promoción y publicidad del almacén, a fin de incentivar la adquisición de los productos. Finalmente se realizó el análisis financiero, el mismo que permitió determinar técnicamente los beneficios económicos que generan la implementación del plan de marketing, asegurar de esta manera el aprovechamiento de las oportunidades comerciales de las empresas en el mercado. El desarrollo del trabajo investigativo se realizó mediante el método inductivo, ya que se basa en el razonamiento para poder obtener las conclusiones, el análisis el mismo además se utilizó el método estadístico para el procesamiento de la información, las pequeñas y medianas empresas pymes, contribuyen cada vez más en forma significativa a la economía local, regional y nacional, no solo en términos de rublos generado sino también por el elemento humano que involucra generando empleo, debido al mercado globalizado en que se encuentran inmersas las pymes, estamos convocados a contribuir en este cambio empresarial en pos de la competitividad internacional del país.

Las pequeñas y medianas empresas cumplen un importante papel en la economía de todos los países, en lo cual las empresas pymes son agentes con cultura, interés y con un espíritu de emprendedores con el fin de sacar adelante su empresa, con el propósito al alcanzar las metas que se propone cumpliendo con su deber satisfacer todas las necesidades del cliente y que su ente sea superior de las competencias.

\section{Conclusiones.}

Las estrategias del plan marketing compuesto por folletos, una valla publicitaria, donde la empresa desarrollara actividades comerciales logrando cumplir de esta manera con unos de los objetivos del plan de marketing.

El estudio de técnico permitió determinar que la microempresa de elaboración y comercialización de un plan de marketing que permita aprovechar las potencialidades de sus recursos.

En el trabajo investigativo se obtuvo el plan de marketing se creara una variedad de instrumento que permitirá a la empresa generar publicidad.

Las grandes diferentes entre las grandes empresas que utilizan las nuevas tecnologías dentro de la economía que identifiquen las necesidades de los clientes en una empresa. 


\section{Referencia Bibliográfica.}

ALCAIDE, Juan; BERNUÉS, Sergio; DÍAZ, Esmeralda; ESPINOZA, Roberto; MUÑIZ, Rafael; SMITH, Christopher. Marketing y PYMES. Primera Edición. 2013. ISBN: 97884-695-7487-4.

CAMPOY, Daniel. Como Gestionar y Planificar un Proyecto en la Empresa. Primera Edición. España. Editorial Ideas propias. 2009. ISBN: 978-84-9839-168-8.

ESCUDERO, María. Gestión Comercial y Servicio de Atención al Cliente. Primera Edición. España. Ediciones Paraninfo, S.A. 2011. ISBN: 978-84-9732-851-7.

FERNÁNDEZ, Ricardo. La Mejora de la Productividad en la Pequeña y Mediana Empresa. Primera Edición. España. Editorial Club Universitario. 2010. ISBN: 978-848454-978-9.

LAMBIN, Charles; HAIR, Joshed; CARL, McDaniel. Marketing. Primera Edición. México. Cangare Liaoning Editores, S.A. 2009. ISBN: 978-607-481-519-1.

MUNUERA, José L.; RODRIGUEZ, Ana I. Estrategias de Marketing. Segunda Edición. España. Editorial ESIC. 2012. ISBN: 978-84-7356-819-7.

Blas Jiménez M. (2013), Diccionario de Administración y Finanzas, Editorial Palibrio, Primera edición, Estados Unidos, N. ${ }^{\circ}$ de páginas 594, ISBN: 1463354959,.

Briseño Ramírez H. (2010), Indicadores Financieros, Ediciones Umbral, Primera edición, México, ISBN: 9789709758405.

Escudero Serrano M. (2014), Gestión de compras Comercio y marketing, Ediciones Paraninfo, Primera edición, Madrid, N. º de páginas 284, ISBN: 8428333696.

Herrera A. José A. (2012), Administración de La Empresa Constructora, Editorial Lulu.com, Primera edición, Madrid, ISBN: 130034162, Nº de paginas 160

ARTAL Manuel, (2013), Dirección de ventas Organización del departamento de ventas y gestión de vendedores, Duodécima edición, Esic editorial, Madrid, ISBN: 8473569741,9788473569743 .

AGUEDA Esteban, MOLINA Arturo, (2014), Fundamentos del Marketing, Esic editorial, Segunda edición, Madrid, ISBN 8473568915, 9788473568913, Nº de páginas 210.

BAHILlO María, María Pérez, (2010), Gestión de la documentación jurídica y empresarial, Ediciones Paraninfo, Segunda Edición, España, ISBN: 8428335605, 9788428335607, N. ${ }^{\circ}$ de páginas 380. 
DÍAZ Cesar, GONZÁLEZ Roberto, LÓPEZ Flavio, PRECIADO Claudia, (2013), Mercadotecnia digital y publicidad online, Editorial Universidad de Guadalajara, Primera edición, México, ISBN: 6074509026, 9786074509021, N. ${ }^{\circ}$ de páginas 112.

\section{Para citar el artículo indexado.}

Velasteguí E, \& Guamangate M. (2017). Marketing Publicitario Mipymes. Revista electrónica Visionario Digital 1(1), 19-33. Recuperado desde: http://cienciadigital.org/revistacienciadigital2/index.php/VisionarioDigital/article/view/ $\underline{255 / 559}$

\section{LCiencia}

El artículo que se publica es de exclusiva responsabilidad de los autores y no necesariamente reflejan el pensamiento de la Revista Ciencia Digital.

El articulo queda en propiedad de la revista y, por tanto, su publicación parcial y/o total en otro medio tiene que ser autorizado por el director de la Revista Ciencia Digital
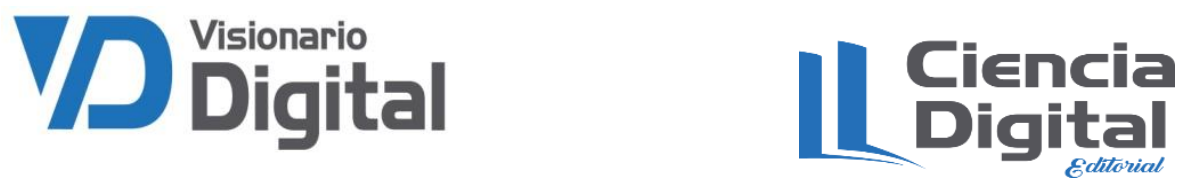